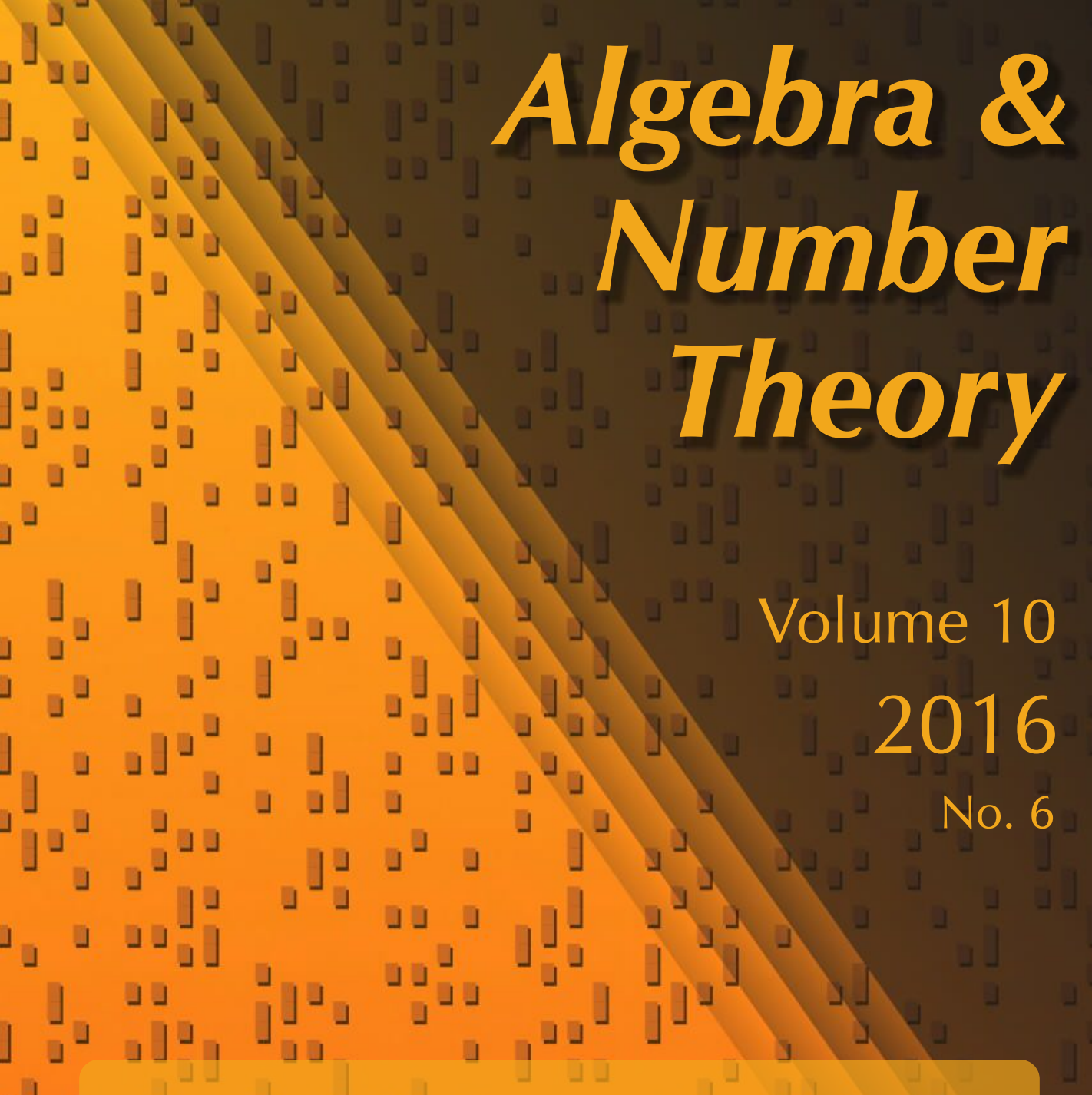

A probabilistic Tits alternative and probabilistic identities

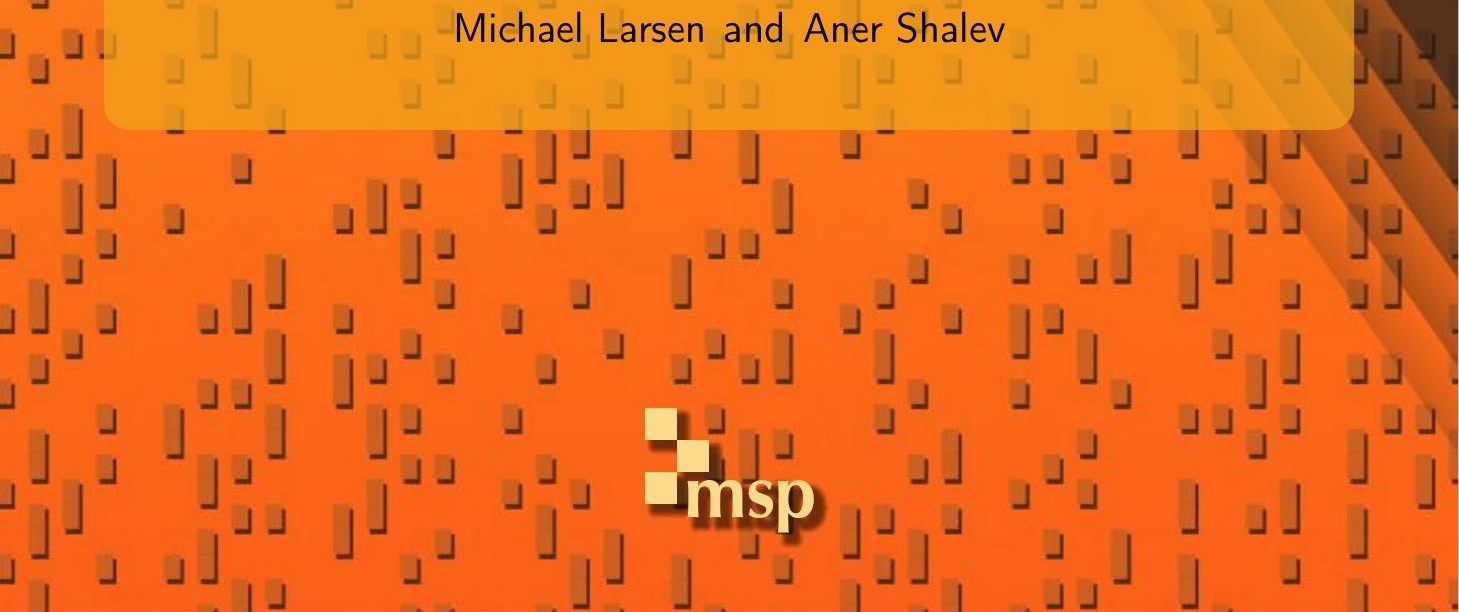




\title{
A probabilistic Tits alternative and probabilistic identities
}

\author{
Michael Larsen and Aner Shalev
}

\begin{abstract}
We introduce the notion of a probabilistic identity of a residually finite group $\Gamma$. By this we mean a nontrivial word $w$ such that the probabilities that $w=1$ in the finite quotients of $\Gamma$ are bounded away from zero.

We prove that a finitely generated linear group satisfies a probabilistic identity if and only if it is virtually solvable.

A main application of this result is a probabilistic variant of the Tits alternative: Let $\Gamma$ be a finitely generated linear group over any field and let $G$ be its profinite completion. Then either $\Gamma$ is virtually solvable, or, for any $n \geq 1, n$ random elements $g_{1}, \ldots, g_{n}$ of $G$ freely generate a free (abstract) subgroup of $G$ with probability 1 .
\end{abstract}

We also prove other related results and discuss open problems and applications.

\section{Introduction}

The celebrated Tits alternative [1972] asserts that a finitely generated linear group is either virtually solvable or has a (nonabelian) free subgroup. A number of variations and extensions of this result have been obtained over the years. In particular, it is shown in [Breuillard and Gelander 2007] that if $\Gamma$ is a finitely generated linear group which is not virtually solvable then its profinite completion $\widehat{\Gamma}$ has a dense free subgroup of finite rank (this answers a question from [Dixon et al. 2003], where a somewhat weaker result was obtained). The purpose of this paper is to establish a probabilistic version of the Tits alternative, and to relate it to the notion of probabilistic identities, which is interesting in its own right.

In order to formulate our first result, let us say that a profinite group $G$ is randomly free if for any positive integer $n$ the set of $n$-tuples in $G^{n}$ which freely generate a free subgroup of $G$ (isomorphic to $F_{n}$ ) has measure 1 (with respect to the normalized

Larsen was partially supported by NSF grant DMS-1401419. Shalev was partially supported by ERC advanced grant 247034, ISF grant 1117/13 and the Vinik Chair of Mathematics, which he holds. MSC2010: primary 20G15; secondary 20E18.

Keywords: Tits alternative, residually finite, virtually solvable, probabilistic identity, profinite completion. 
Haar measure on $G^{n}$ ). We also say that a (discrete) residually finite group $\Gamma$ is randomly free if its profinite completion is randomly free.

Recall that related notions have already been studied in various contexts. For example, Epstein [1971] showed that connected finite-dimensional nonsolvable real Lie groups are randomly free (in the sense that the set of $n$-tuples which do not freely generate a free subgroup has measure zero). Later it was shown by Szegedy [2005] that the Nottingham pro- $p$ group is randomly free (answering a question of the second author). Furthermore, Abert proved [2005] that some other groups are randomly free; these include the Grigorchuk group and profinite weakly branch groups.

We can now state our probabilistic Tits alternative.

Theorem 1.1. Let $\Gamma$ be a finitely generated linear group over any field. Then either $\Gamma$ is virtually solvable or $\Gamma$ is randomly free.

The proof of this result relies on the notion and properties of probabilistic identities which we introduce below.

Let $w=w\left(x_{1}, \ldots, x_{n}\right)$ be a nontrivial element of the free group $F_{n}$, and let $\Gamma$ be a residually finite group. Consider the induced word map $\Gamma^{n} \rightarrow \Gamma$, which, by a slight abuse of notation, we also denote $w$. If the image $w\left(\Gamma^{n}\right)$ of this map is $\{1\}$ then $w$ is an identity of $\Gamma$. We say that $w$ is a probabilistic identity of $\Gamma$ if there exists $\epsilon>0$ such that, for each finite quotient $H=\Gamma / \Delta$ of $\Gamma$, the probability $P_{H}(w)$ that $w\left(h_{1}, \ldots, h_{n}\right)=1$ (where the $h_{i} \in H$ are chosen independently with respect to the uniform distribution on $H$ ) is at least $\epsilon$. This amounts to saying that, in the profinite completion $G=\widehat{\Gamma}$ of $\Gamma$, the probability (with respect to the Haar measure) that $w\left(g_{1}, \ldots, g_{n}\right)=1$ is positive.

For example, $w=x_{1}^{2}$ is a probabilistic identity of the infinite dihedral group $\Gamma=D_{\infty}$, since in any finite quotient $\Gamma / \Delta=D_{n}$ of $\Gamma$ we have $P_{\Gamma / \Delta}(w) \geq \frac{1}{2}$. Note that, in this example, $w$ is not an identity on a finite index subgroup of $\Gamma$, but it is an identity on a coset of the cyclic subgroup of index two.

More generally, probabilistic identities may be regarded as an extension of the notion of coset identities. Recall that a word $1 \neq w \in F_{n}$ is said to be a coset identity of the infinite group $\Gamma$ if there exists a finite index subgroup $\Delta \leq \Gamma$ and cosets $\gamma_{1} \Delta, \ldots, \gamma_{n} \Delta$ (where $\left.\gamma_{i} \in \Gamma\right)$ such that $w\left(\gamma_{1} \Delta, \ldots, \gamma_{n} \Delta\right)=\{1\}$.

Our main result on probabilistic identities is the following.

Theorem 1.2. A finitely generated linear group satisfies a probabilistic identity if and only if it is virtually solvable.

Theorem 1.2 has several consequences. First, it easily implies Theorem 1.1. To show this, suppose $\Gamma$ is not virtually solvable, and let $G$ be the profinite completion of $\Gamma$. Note that $g_{1}, \ldots, g_{n} \in G$ freely generate a free subgroup of $G$ if and only if $w\left(g_{1}, \ldots, g_{n}\right) \neq 1$ for every $1 \neq w \in F_{n}$. By Theorem 1.2 above, the probability 
that $w\left(g_{1}, \ldots, g_{n}\right)=1$ is 0 for any such $w$. As Haar measure is $\sigma$-additive, the probability that there exists $w \neq 1$ such that $w\left(g_{1}, \ldots, g_{n}\right)=1$ is also 0 . Thus, $g_{1}, \ldots, g_{n}$ freely generate a free subgroup with probability 1 , proving Theorem 1.1.

Secondly, Theorem 1.2 immediately implies the following.

Corollary 1.3. A finitely generated linear group which satisfies a probabilistic identity satisfies an identity.

It would be interesting to find out whether the same holds without the linearity assumption. We discuss this and related problems and applications in Section 3.

In the course of the proof of Theorem 1.2 we establish a result of independent interest, showing that probabilistic identities on finitely generated linear groups are in fact coset identities.

The arguments proving this result also prove a more general result on probabilistic identities with parameters. Let $w\left(x_{1}, \ldots, x_{n}, y_{1}, \ldots, y_{m}\right)$ be a word in the variables $x_{1}, \ldots, x_{n}, y_{1}, \ldots, y_{m}$, and let $\gamma_{1}, \ldots, \gamma_{m}$ be elements of a residually finite group $\Gamma$. Consider the word with parameters $v\left(x_{1}, \ldots, x_{n}\right):=w\left(x_{1}, \ldots, x_{n}, \gamma_{1}, \ldots, \gamma_{m}\right)$. The notions of a probabilistic identity with parameters and of a coset identity with parameters are then defined in the obvious way.

Note that Theorem 1.2 cannot be generalized to probabilistic identities with parameters. For example, let $\gamma_{1} \in \Gamma$ be a central element. Then the word with parameters $\left[x_{1}, \gamma_{1}\right]$ is an identity on $\Gamma$, though $\Gamma$ need not be virtually solvable. However, we can show the following.

Theorem 1.4. Let $\Gamma$ be a finitely generated linear group over any field. Then every probabilistic identity (possibly with parameters) on $\Gamma$ is a coset identity.

It easily follows that, if $w$ is a word in $n$ variables (possibly with parameters from $\Gamma$ ), and $\gamma \in \Gamma$ is such that in all finite quotients $H=\Gamma / \Delta$ of $\Gamma$ the probability that $w\left(h_{1}, \ldots, h_{n}\right)=\gamma+\Delta$ is at least some fixed $\epsilon>0$, then the fiber $w^{-1}(\gamma)$ contains the Cartesian product $\gamma_{1} \Delta \times \cdots \times \gamma_{n} \Delta$ of cosets of some finite index subgroup $\Delta \leq \Gamma$. Indeed, apply Theorem 1.4 to the word with parameters $w \gamma^{-1}$.

In fact, the proof of Theorem 1.4 gives rise to an even more general result of independent interest. In order to formulate it, let $\Gamma$ be a linear group and let $n$ be a positive integer. Let us say that a subset $\Xi$ of $\Gamma^{n}$ is Zariski-closed if there is an embedding of $\Gamma$ in $\mathrm{GL}_{r}(F)$ (for some field $F$ and a positive integer $r$ ) and a Zariski-closed subset $X$ of $\mathrm{GL}_{r}^{n}$ such that $\Xi=X(F) \cap \Gamma^{n}$.

Then we have the following.

Theorem 1.5. Let $\Gamma$ be a finitely generated linear group over any field, and let $n \geq 1$. Let $\Xi \subseteq \Gamma^{n}$ be a Zariski-closed subset. Suppose there exists $\epsilon>0$ such that $\left|\Xi \Delta^{n} / \Delta^{n}\right| \geq \epsilon|\Gamma / \Delta|^{n}$ for all normal subgroups of finite index $\Delta$ of $\Gamma$. Then there exists a finite index subgroup $\Delta \leq \Gamma$ and elements $\gamma_{1}, \ldots, \gamma_{n} \in \Gamma$ such that $\Xi \supseteq \gamma_{1} \Delta \times \cdots \times \gamma_{n} \Delta$. 
This result is proved using an easy adaptation of the proof of Theorem 1.4, which we leave for the interested reader. Theorem 1.5 amounts to saying that if the closure of $\Xi$ in the profinite group $(\widehat{\Gamma})^{n}$ has positive Haar measure, then it has a nonempty interior.

It is shown in [Breuillard and Gelander 2007, Theorem 8.4] that a finitely generated linear group which satisfies a coset identity (without parameters) is virtually solvable. Using this result we can immediately deduce Theorem 1.2 from Theorem 1.4. In fact, we provide here a self-contained proof of Theorem $1.2 \mathrm{using}$ Theorem 1.4 and Proposition 2.5 below.

Our original approach to proving Theorem 1.2 relied on strong approximation for linear groups and on establishing upper bounds on the probabilities $P_{G}(w)$, where $G$ is a group satisfying $T^{k} \leq G \leq \operatorname{Aut}\left(T^{k}\right)$ for a finite simple group $T$. However, this approach is rather involved. A shorter and simpler proof of Theorems 1.4 and 1.2 is given in Section 2.

The idea is to use linearity to map $\Gamma$ into a "linear algebraic group" $G$ over an infinite product $\prod_{\mathfrak{m}} A / \mathfrak{m}$ of finite fields. The closure of the image is then a profinite group. Suppose that for some Zariski-closed subset $X \subset G^{n}$, the measure of the closure of $X\left(\prod_{\mathfrak{m}} A / \mathfrak{m}\right) \cap \Gamma^{n}$ is positive. Every translate of $X$ by an element of $\Gamma^{n}$ has the same property. Unless $X$ is a union of connected components of $G^{n}$ we can find an infinite set of pairwise distinct translates of $X$, each of which has the same positive-measure property. Thus, some pairs of translates of $X$ must intersect $\Gamma^{n}$ with positive measure; intersecting $X$ with a suitable translate by an element of $\Gamma^{n}$, we obtain a proper closed subset of $X$ with the same property as $X$ itself. This process cannot continue indefinitely. The theorem is obtained by applying it to the fiber over 1 of a nontrivial word map $w$. The actual implementation uses the language of (affine) schemes and a notion somewhat weaker than that of measure.

In fact, this method of proof, and Proposition 2.5 in particular, yields the following extension of Theorem 1.2: Suppose $\Gamma$ is a finitely generated linear group which is not virtually solvable. Then all fibers in $(\widehat{\Gamma})^{n}$ of all nontrivial words $w \in F_{n}$ have measure 0.

In other words, for a finite group $H$, let $P_{H, w}$ denote the probability distribution induced on $H$ by $w$ (so that, for $h \in H, P_{w, H}(h)$ is the probability that $\left.w\left(h_{1}, \ldots, h_{n}\right)=h\right)$. Its $\ell_{\infty}$-norm is defined by $\left\|P_{H, w}\right\|_{\infty}=\max _{h \in H} P_{H, w}(h)$. Then we have:

Theorem 1.6. Let $\Gamma$ be a finitely generated linear group. Suppose for some $n \geq 1$ and $1 \neq w \in F_{n}$ there exists $\epsilon>0$ such that for all finite quotients $H$ of $\Gamma$ we have $\left\|P_{H, w}\right\|_{\infty} \geq \epsilon$. Then $\Gamma$ is virtually solvable.

See also [Aoun 2011] for a different probabilistic Tits alternative, related to certain random walks on the discrete linear group $\Gamma$. 


\section{Proof of Theorems 1.4 and 1.2}

If a group $\Gamma$ acts on a topological space $X$ and $Y \subseteq X$, we say $Y$ is $\Gamma$-finite if its orbit under $\Gamma$ is finite. We say a closed subset $Z \subseteq X$ is $\Gamma$-covered by $Y$ if $Z$ is a closed subset of some finite union of $\Gamma$-translates of $Y$.

Lemma 2.1. Let $\Gamma$ be a group acting on a set $X$. If $Y_{1}, \ldots, Y_{n}$ are subsets of $X$ which are not $\Gamma$-finite, then there exists $g \in \Gamma$ such that $g Y_{i} \neq Y_{j}$ for $1 \leq i, j \leq n$.

Proof. For given $i, j$, the set of $g$ such that $g Y_{i}=Y_{j}$ is either empty or is a left coset of the stabilizer of $Y_{i}$ in $\Gamma$. By a theorem of B. H. Neumann [1954], a group cannot be covered by a finite collection of left cosets of subgroups of infinite index. The result follows.

Proposition 2.2. Let $X$ be a Noetherian topological space and $\Gamma$ a group of homeomorphisms $X \rightarrow X$. Let $f$ denote a function from the set of closed subsets of $X$ to $[0,1]$ satisfying the following conditions:

(I) If $Z \subseteq Y$ are closed subsets of $X$, then $f(Z) \leq f(Y)$.

(II) For all closed subsets $Y \subseteq X$ and all $g \in \Gamma$ such that $f(Y \cap g Y)=0$, we have

$$
f(Y \cup g Y) \geq 2 f(Y) .
$$

If $Y \subseteq X$ is closed and $\Gamma$-covers some closed subset $W \subseteq X$ with $f(W)>0$, then $Y$ $\Gamma$-covers some closed $\Gamma$-stable subset $Z \subseteq X$ with $f(Z)>0$.

Proof. By the Noetherian hypothesis, we may assume without loss of generality that $Y$ is minimal for the property of $\Gamma$-covering a set of positive $f$-value. If two distinct irreducible components $Y_{i}$ and $Y_{j}$ of $Y$ were $\Gamma$-translates of one another, we could replace $Y$ with the union of all of its components except $Y_{j}$, and the resulting closed set would still $\Gamma$-cover a set of positive $f$-value. This is impossible by the minimality of $Y$.

If $Y$ is $\Gamma$-finite, then

$$
Z:=\bigcup_{g \in \Gamma} g Y
$$

is a $\Gamma$-stable finite union of $\Gamma$-translates of $Y$ containing $W$. By condition (I), it satisfies $f(Z)>0$, so we are done. As $Y$ is a finite union of irreducible components, we may therefore assume at least one such component $Y_{0}$ is not $\Gamma$-finite. We write $Y=Y_{0} \cup Y^{\prime}$, where no $\Gamma$-translate of $Y^{\prime}$ contains $Y_{0}$.

By condition (I), there exists a finite sequence $g_{1}, \ldots, g_{r} \in \Gamma$ such that $f(Z)>0$ for

$$
Z:=g_{1} Y \cup \cdots \cup g_{r} Y
$$


We choose the $g_{i}$ so that

$$
f(Z)>\frac{\sup _{\Delta \subsetneq \Gamma \text { finite }} f\left(\bigcup_{g \in \Delta} g Y\right)}{2} .
$$

As no $\Gamma$-translate of $Y_{0}$ is $\Gamma$-finite, Lemma 2.1 implies that there exists $g$ such that $g_{i} Y_{0} \neq g g_{j} Y_{0}$ for all $i, j$. Thus,

$$
Y^{\prime} \cup \bigcup_{i, j}\left(Y_{0} \cap g_{i}^{-1} g g_{j} Y_{0}\right) \subsetneq Y
$$

$\Gamma$-covers $Z \cap g Z$. By the minimality of $Y$, this means $f(Z \cap g Z)=0$. By condition (II), $f(Z \cup g Z) \geq 2 f(Z)$, which contradicts (2-1). We conclude that $Z$ must be $\Gamma$-finite.

Now, let $A$ be an integral domain finitely generated over $\mathbb{Z}$ with fraction field $K$. Let $\mathcal{G}=\operatorname{Spec} B$ be an affine group scheme of finite type over $A$ (see [Waterhouse 1979]). As usual, for every commutative $A$-algebra $T$, let $\mathcal{G}(T)$ denote the set of Spec $T$-points of $\mathcal{G} \rightarrow \operatorname{Spec} A$, i.e., the set of $A$-algebra homomorphisms $B \rightarrow T$. The group structure on $\mathcal{G}$ makes each $\mathcal{G}(T)$ a group, functorially in $T$. We regard $\mathcal{G}$ as a topological space with respect to its Zariski topology. If $Y \subseteq \mathcal{G}$ is a closed subset, we define $Y(T)$ to be the subset of $\mathcal{G}(T)$ consisting of $A$-homomorphisms $B \rightarrow T$ such that the corresponding map of topological spaces Spec $T \rightarrow \mathcal{G}$ sends Spec $T$ into a subset of $Y$. If $Z \subseteq \mathcal{G}$ is another closed subset, then

$$
(Y \cap Z)(T)=Y(T) \cap Z(T),
$$

but, in general, the inclusion

$$
Y(T) \cup Z(T) \subseteq(Y \cup Z)(T)
$$

need not be an equality.

We define

$$
P(\mathcal{G}, A):=\prod_{\mathfrak{m} \in \operatorname{Maxspec}(A)} \mathcal{G}(A / \mathfrak{m}),
$$

where Maxspec denotes the set of maximal ideals, and $P(\mathcal{G}, A)$ is endowed with the product topology. Note that as $\mathcal{G}$ is of finite type (i.e., $B$ is a finitely generated $A$-algebra) and every $A / \mathfrak{m}$ is a field finitely generated over $\mathbb{Z}$ (and hence finite), it follows that each $\mathcal{G}(A / \mathfrak{m})$ is finite and $P(\mathcal{G}, A)$ is a profinite group. For any closed subset $X \subseteq \mathcal{G}$, we define the closed subset

$$
P(X, A):=\prod_{\mathfrak{m} \in \operatorname{Maxspec}(A)} X(A / \mathfrak{m}) \subseteq P(\mathcal{G}, A) .
$$

Lemma 2.3. If $X \subseteq \mathcal{G}$ does not meet the generic fiber $\operatorname{Spec} B \otimes_{A} K \subset \mathcal{G}$, then $P(X, A)$ is empty. 
Proof. If $I \subseteq B$ is the ideal defining $X$, then $(B / I) \otimes_{A} K=0$, so $I \otimes_{A} K=B \otimes_{A} K$. It follows that there exist elements $b_{i} \in I$ and $a_{i} / a_{i}^{\prime} \in K$ such that

$$
\sum_{i} b_{i} \otimes \frac{a_{i}}{a_{i}^{\prime}}=1
$$

and clearing denominators we see that some nonzero element $a^{\prime}:=\prod_{i} a_{i}^{\prime} \in A$ belongs to $I$. If $\mathfrak{m}$ is a maximal ideal of $A\left[1 / a^{\prime}\right]$, then $A\left[1 / a^{\prime}\right] / \mathfrak{m}$ is a field finitely generated over $\mathbb{Z}$, hence a finite field, and therefore $\mathfrak{m} \cap A$ is a maximal ideal of $A$. Thus, the image of $a^{\prime}$ in $A /(\mathfrak{m} \cap A)$ is nonzero, from which it follows that there are no $A$-homomorphisms $B / I \rightarrow A /(\mathfrak{m} \cap A)$, i.e., $X(A /(\mathfrak{m} \cap A))=\varnothing$.

For any subgroup $\Gamma \subseteq \mathcal{G}(A) \subseteq P(\mathcal{G}, A)$, we define $\bar{\Gamma}$ to be the closure of $\Gamma$ in $P(\mathcal{G}, A)$. This is a closed subgroup of a profinite group and therefore a profinite group itself. We endow it with Haar measure $\mu_{\bar{\Gamma}}$, normalized so that $\left(\bar{\Gamma}, \mu_{\bar{\Gamma}}\right)$ is a probability space. In particular, left translation by $\Gamma$ is a continuous measurepreserving action on $\left(\bar{\Gamma}, \mu_{\bar{\Gamma}}\right)$. As Haar measure is outer regular, for every Borel set $B$,

$$
\mu_{\bar{\Gamma}}(B)=\inf _{S \subseteq \operatorname{Maxspec}(A)} \frac{\left|\mathrm{pr}_{S} B\right|}{\left|\operatorname{pr}_{S} \Gamma\right|},
$$

where $S$ ranges over all finite sets of maximal ideals of $A$ and $\operatorname{pr}_{S}$ denotes projection onto $\prod_{\mathfrak{m} \in S} \mathcal{G}(A / \mathfrak{m})$.

For any positive integer $n$, we let $\mathcal{G}^{n}$ denote the $n$-th fiber power of $\mathcal{G}$ relative to $A$, i.e., defining

$$
B_{n}:=\underbrace{B \otimes_{A} B \otimes_{A} \cdots \otimes_{A} B}_{n}
$$

we define $\mathcal{G}^{n}:=$ Spec $B_{n}$, regarded as a topological space with respect to the Zariski topology. Note that in general the Zariski topology on $\mathcal{G}^{n}$ is not the product topology. However, by the universal property of tensor products, $\mathcal{G}^{n}(T)$ is canonically isomorphic to $\mathcal{G}(T)^{n}$ for all commutative $A$-algebras $T$. Moreover, $B_{n}$ is a finitely generated $\mathbb{Z}$-algebra, and by the Hilbert basis theorem this implies that $\mathcal{G}^{n}$ is a Noetherian topological space.

We consider the closure $\bar{\Gamma}^{n}$ of $\Gamma^{n}$ in $P\left(\mathcal{G}^{n}, A\right)$. For any closed subset $Y \subseteq \mathcal{G}^{n}$, we define

$$
P_{\Gamma}(Y):=\bar{\Gamma}^{n} \cap P(Y, A) .
$$

Thus, if $Y$ and $Z$ are closed subsets of $\mathcal{G}^{n}$,

$$
P_{\Gamma}(Y \cap Z)=\bar{\Gamma}^{n} \cap P(Y \cap Z, A)=\bar{\Gamma}^{n} \cap(P(Y, A) \cap P(Z, A))=P_{\Gamma}(Y) \cap P_{\Gamma}(Z) .
$$


As

$$
P(Y \cup Z, A)=\prod_{\mathfrak{m} \in \operatorname{Maxspec}(A)}(Y(A / \mathfrak{m}) \cup Z(A / \mathfrak{m})) \supseteq P(Y, A) \cup P(Z, A),
$$

we have

$$
P_{\Gamma}(Y \cup Z) \supseteq P_{\Gamma}(Y) \cup P_{\Gamma}(Z) .
$$

Defining

$$
f(Y):=\mu_{\bar{\Gamma}^{n}}\left(P_{\Gamma}(Y)\right),
$$

condition (I) of Proposition 2.2 is obvious. As $\mu_{\bar{\Gamma}^{n}}$ is a measure, if $f(Y \cap Z)=0$, then

$$
\begin{aligned}
f(Y \cup Z) & =\mu_{\bar{\Gamma}^{n}}\left(P_{\Gamma}(Y \cup Z)\right) \\
& \geq \mu_{\bar{\Gamma}^{n}}\left(P_{\Gamma}(Y) \cup P_{\Gamma}(Z)\right) \\
& =\mu_{\bar{\Gamma}^{n}}\left(P_{\Gamma}(Y)\right)+\mu_{\bar{\Gamma}^{n}}\left(P_{\Gamma}(Z)\right)-\mu_{\bar{\Gamma}^{n}}\left(P_{\Gamma}(Y) \cap P_{\Gamma}(Z)\right) \\
& =f(Y)+f(Z)-f(Y \cap Z)=f(Y)+f(Z) .
\end{aligned}
$$

As $\mu_{\bar{\Gamma}^{n}}$ is $\Gamma^{n}$-invariant, this implies condition (II).

Proposition 2.4. Let $G$ denote a linear algebraic group over a field $K$. If $\Gamma$ is Zariski-dense in $G(K)$, then a nonempty closed subset $Y$ of $G^{n}$ is $\Gamma^{n}$-finite if and only if it is a union of connected components of $G^{n}$.

Proof. If $Y$ is $\Gamma^{n}$-finite, its stabilizer $\Delta$ is of finite index in $\Gamma^{n}$, which implies that the Zariski closure $D$ of $\Delta$ in $G^{n}$ has finite index in $G^{n}$. Thus $D \cap\left(G^{n}\right)^{\circ}$ is of finite index in $\left(G^{n}\right)^{\circ}$. As $\left(G^{n}\right)^{\circ}$ is connected, it follows that $D$ contains $\left(G^{n}\right)^{\circ}$. The Zariski closure of any left coset of $\Gamma^{n}$ is a left coset of $D$ and therefore a union of cosets of $\left(G^{n}\right)^{\circ}$. Conversely, any left translate of a coset of $\left(G^{n}\right)^{\circ}$ is again such a coset, so the orbit of any union of connected components of $G^{n}$ is finite.

We can now prove Theorem 1.4.

Proof. We fix a faithful representation $\rho: \Gamma \rightarrow \mathrm{GL}_{r}(F)$, where $F$ is an algebraically closed field. Let $G \subset \mathrm{GL}_{r}$ denote the Zariski closure of $\Gamma$ in $\mathrm{GL}_{r}$.

We recall how to extend $G$ to a subgroup scheme of $\mathrm{GL}_{r}$ defined over a finitely generated $\mathbb{Z}$-algebra. Let

$$
R_{\mathbb{Z}, r}:=\mathbb{Z}\left[x_{i j}, y\right]_{i, j=1, \ldots, r} /\left(y \operatorname{det}\left(x_{i j}\right)-1\right)
$$

denote the coordinate ring of $\mathrm{GL}_{r}$ over $\mathbb{Z}$, and let

$$
\Delta_{\mathbb{Z}, r}: R_{\mathbb{Z}, r} \rightarrow R_{\mathbb{Z}, r} \otimes_{\mathbb{Z}} R_{\mathbb{Z}, r}, \quad S_{\mathbb{Z}, r}: R_{\mathbb{Z}, r} \rightarrow R_{\mathbb{Z}, r}, \quad \text { and } \quad \epsilon_{\mathbb{Z}, r}: R_{\mathbb{Z}, r} \rightarrow \mathbb{Z}
$$

denote the ring homomorphisms associated to the multiplication, inverse, and unit maps. Closed subschemes of $\mathrm{GL}_{r}$ over any commutative ring $A$ are in one-to-one 
correspondence with ideals $I$ of $R_{A, r}:=A \otimes_{\mathbb{Z}} R_{\mathbb{Z}, r}$, and such an ideal defines a group subscheme if and only if $I$ is a Hopf ideal [Waterhouse 1979, §2.1], i.e., if and only if it satisfies the following three conditions:

$$
\begin{aligned}
\Delta_{A, r}(I) & \subseteq I \otimes_{A} R_{A, r}+R_{A, r} \otimes_{A} I, \\
S_{A, r}(I) & \subseteq I, \\
\epsilon_{A, r}(I) & =\{0\} .
\end{aligned}
$$

We fix a finite set of generators $h_{k}$ of the ideal $I_{F}$ in $R_{F, r}$ associated to $G$ as a closed subvariety of $\mathrm{GL}_{r}$ over $F$. We lift each $h_{k}$ to an element $\tilde{h}_{k} \in F\left[x_{i j}, y\right]$. For any subring $A \subseteq F$ such that $\tilde{h}_{k} \in A\left[x_{i j}, y\right]$, we denote again by $h_{k}$ the image of $\tilde{h}_{k}$ in $R_{A, r}$; this should not cause confusion. Let $A_{0}$ denote the subring of $F$ generated by all matrix entries in $\mathrm{GL}_{r}(F)$ of the $\rho\left(g_{j}\right)$, as $g_{j}$ runs over some finite generating set of $\Gamma$, together with all coefficients of the $\tilde{h}_{k}$. Let $I_{0}$ denote the ideal generated by the elements $h_{k}$ in $R_{A_{0}, r}$, and let $K$ denote the fraction field of $A_{0}$. As

$$
\Delta_{A_{0}, r}\left(I_{0}\right) \subseteq I_{0} \otimes_{A_{0}} R_{K, r}+R_{K, r} \otimes_{A_{0}} I_{0}
$$

and

$$
S_{A_{0}, r}\left(I_{0}\right) \subseteq I_{0} \otimes_{A_{0}} R_{K, r},
$$

there exists $a \in A_{0}$ such that

$$
\Delta_{A_{0}, r}\left(h_{i}\right) \in I_{0} \otimes_{A_{0}} R_{A_{0}[1 / a], r}+R_{A_{0}[1 / a], r} \otimes_{A_{0}} I_{0}
$$

and

$$
S_{A_{0}, r}\left(h_{i}\right) \in I_{0} \otimes_{A_{0}} A_{0}[1 / a]
$$

for all $i$, and therefore, setting $A:=A_{0}[1 / a]$ and $I:=I_{0} \otimes_{A_{0}} A$, we have that $I$ is a Hopf ideal of $R_{A, r}$. We set $\mathcal{G}:=\operatorname{Spec} R_{A, r} / I$, the closed group subscheme of $\mathrm{GL}_{r}$ over $A$ defined by $h_{k} \in R_{A, r}$. By construction, $\rho(\Gamma)$ is a Zariski-dense finitely generated subgroup of $\mathcal{G}(A)$.

Now, let $w$ be a probabilistic identity on $\Gamma$ (possibly with parameters). Consider $w$ as a morphism of schemes over $A$ from $\mathcal{G}^{n}$ to $\mathcal{G}$. Let $Y:=w^{-1}(1) \subseteq \mathcal{G}^{n}$. We define $f$ as above. If $f(Y)>0$, then $Y \Gamma$-covers a set of positive $f$-value, so by Proposition $2.2 Y \Gamma$-covers a closed $\Gamma$-stable subset $Z$ with $f(Z)>0$. By Lemma 2.3, $Z$ must meet the generic fiber $G^{n}$ of $\mathcal{G}^{n}$, which implies that $Y$ must meet the generic fiber. Proposition 2.4 now implies that $Z \cap G^{n}$ contains a connected component of $G^{n}$, and it follows that $Y \cap G^{n}$ contains a connected component, i.e., $w$ is a coset identity. Thus, we may assume $f(Y)=0$.

Therefore, for every $\epsilon>0$, there exists a finite set $S$ of maximal ideals of $A$ such that

$$
\frac{\left|\mathrm{pr}_{S} w^{-1}(1)\right|}{\left|\mathrm{pr}_{S} \Gamma^{n}\right|}<\epsilon
$$


Defining $\Delta$ to be the kernel of $\mathrm{pr}_{S}$, we see that, in the finite quotient $\Gamma / \Delta$, the probability that the word map $w$ attains the value $1+\Delta$ is less than $\epsilon$. It follows that $w$ is not a probabilistic identity on $\Gamma$. This contradiction completes the proof of Theorem 1.4.

Proposition 2.5. Let $K$ be a field and $G$ a linear algebraic group over $K$ with nontrivial adjoint semisimple identity component. Let $w \in F_{n}$ be a nontrivial word and let $g_{0} \in G(K)$. Then $w^{-1}\left(g_{0}\right)$ does not contain any connected component of $G^{n}$. Proof. Equivalently, we claim that $\operatorname{dim} w^{-1}\left(g_{0}\right)<\operatorname{dim} G^{n}$. Since dimensions do not depend on the base field, we may and shall assume, without loss of generality, that $K$ is algebraically closed. Let $G^{\circ}$ be the identity component, $T$ a maximal torus of $G^{\circ}$ and $B$ a Borel subgroup of $G^{\circ}$ containing $T$. Let $\Phi$ be the root system of $G$ with respect to $T$, and let $\Phi^{+}$denote the set of roots of $B$ with respect to $T$. Every maximal torus of $G^{\circ}$ is conjugate under $G^{\circ}(K)$ to $T$. The Weyl group $N_{G}(T) / T$ acts transitively on the set of Weyl chambers, so every pair $T^{\prime} \subset B^{\prime}$ is conjugate to $T \subset B$ by some element of $G^{\circ}(K)$. In particular, for any $g \in G(K)$, the pair $g^{-1} T g \subset$ $g^{-1} B g$ is conjugate in $G^{\circ}(K)$ to $T \subset B$, or, equivalently, there is some element $h \in g G^{\circ}(K)$ such that conjugation by $h$ stabilizes $T$ and $B$. The highest root $\alpha$ of $\Phi^{+}$ is determined by $B$, so $h$ likewise preserves $\alpha$. It therefore normalizes $\operatorname{ker} \alpha^{\circ}$, and therefore the derived group $G_{\alpha}$ of the centralizer of $\operatorname{ker} \alpha^{\circ}$. This group is semisimple and of type $A_{1}$, so every element that normalizes it acts by an inner automorphism. It follows that the centralizer of $G_{\alpha}$ in $G$ meets every connected component of $G$.

Suppose now that $w$ is constant on $g_{1} G^{\circ} \times \cdots \times g_{n} G^{\circ}$ for some $g_{1}, \ldots, g_{n} \in G(K)$. Without loss of generality we may assume that all $g_{i}$ centralize $G_{\alpha}$. As $w$ is constant on $g_{1} G_{\alpha} \times \cdots \times g_{n} G_{\alpha}$, and as

$$
w\left(g_{1} h_{1}, \ldots, g_{n} h_{n}\right)=w\left(g_{1}, \ldots, g_{n}\right) w\left(h_{1}, \ldots, h_{n}\right)
$$

for all $h_{1}, \ldots, h_{n} \in G_{\alpha}(K)$, it follows that $w$ is constant on $G_{\alpha}^{n}$. This is impossible because nontrivial words give nontrivial word maps on all semisimple algebraic groups [Borel 1983].

Proof of Theorem 1.2. Every virtually solvable linear group satisfies a nontrivial identity. In the other direction, if $\Gamma \subset \mathrm{GL}_{r}(K)$ satisfies a probabilistic identity, then it satisfies a coset identity by Theorem 1.4, and the same is true for its Zariski closure $G$. If $R$ denotes the maximal solvable normal subgroup of $G^{\circ}$, then $G / R$ also satisfies a coset identity, and by Proposition 2.5 this implies that $G / R$ is finite, i.e., that $G$ is virtually solvable, and so is $\Gamma$.

\section{Open problems}

In this section we discuss related open problems concerning finite and residually finite groups. 
Problem 3.1. Do all finitely generated residually finite groups which satisfy a probabilistic identity satisfy an identity?

We also pose a related, finitary version of Problem 3.1.

Problem 3.2. Is it true that, for any word $1 \neq w \in F_{n}$, any positive integer $d$ and any real number $\epsilon>0$, there exists a word $1 \neq v \in F_{m}$ (for some $m$ ) such that, if $G$ is a finite $d$-generated group satisfying $P_{G}(w) \geq \epsilon$, then $v$ is an identity of $G$ ?

Clearly, a positive answer to Problem 3.2 implies a positive answer to Problem 3.1. Both seem to be very challenging questions, which might have negative answers in general. However, in some special cases they are solved affirmatively. For example, if $w=\left[x_{1}, x_{2}\right]$ or $w=x_{1}^{2}$, then it is known (see [Neumann 1989] and [Mann 1994]) that, for a finite group $G$, if $P_{G}(w) \geq \epsilon>0$, then $G$ is bounded-by-abelian-bybounded (in terms of $\epsilon$ ). This implies affirmative answers to Problems 3.1 and 3.2 for these particular words $w$.

In general we cannot answer these problems for words of the form $x_{1}^{k}(k>2)$. However, for a prime $p$, a result of Khukhro [1986] shows that, if $G$ is a finitely generated pro- $p$ group satisfying a coset identity $x_{1}^{p}$ (namely, there is a coset of an open subgroup consisting of elements of order $p$ or 1 ) then $G$ is virtually nilpotent (and hence satisfies an identity).

Another positive indication is the result showing that for a (nonabelian) finite simple group $T$ and a nontrivial word $w$ we have $P_{T}(w) \rightarrow 0$ as $|T| \rightarrow \infty$ (see [Dixon et al. 2003] for this result, and also [Larsen and Shalev 2012] for upper bounds on $P_{T}(w)$ of the form $\left.|T|^{-\alpha_{w}}\right)$. This implies that a finite simple group $T$ satisfying $P_{T}(w) \geq \epsilon>0$ is of bounded size, hence it satisfies an identity (depending on $w$ and $\epsilon$ only).

Affirmative answers to Problems 3.1 and 3.2 would have far reaching applications. The argument proving Theorem 1.1 above also proves the following.

Proposition 3.3. Assume Problem 3.1 has a positive answer, and let $\Gamma$ be a finitely generated residually finite group. Then either $\Gamma$ satisfies an identity or $\Gamma$ is randomly free.

\section{In particular:}

(i) If $\Gamma$ does not satisfy an identity then $\widehat{\Gamma}$ has a nonabelian free subgroup.

(ii) If $\widehat{\Gamma}$ has a nonabelian free subgroup then almost all $n$-tuples in $\widehat{\Gamma}$ freely generate a free subgroup.

The next application concerns residual properties of free groups. It is well known that the free group $F_{n}$ is residually- $p$. But when is it residually $X$ for a collection $X$ of finite $p$-groups? If this is the case, then $F_{n}$ is also residually $Y$, where $Y$ is the subset of $X$ consisting of $n$-generated $p$-groups. Thus we may replace $X$ by $Y$ and assume all $p$-groups in $X$ are $n$-generated. It is also clear that if $F_{n}(n>1)$ is 
residually $X$ then the groups in $X$ do not satisfy a common identity (namely, they generate the variety of all groups).

It turns out that, assuming an affirmative answer to Problem 3.2, these conditions are also sufficient.

Proposition 3.4. Assume Problem 3.2 has a positive answer. Let $n \geq 2$ be an integer, $p$ a prime, and $X$ a set of $n$-generated finite p-groups. Then the free group $F_{n}$ is residually $X$ if and only if the groups in $X$ do not satisfy a common identity.

To prove this, suppose the groups in $X$ do not satisfy a common identity. To show that $F_{n}$ is residually $X$, we have to find, for each $1 \neq w=w\left(x_{1}, \ldots, x_{n}\right) \in F_{n}$, a group $G \in X$ and an epimorphism $\phi: F_{n} \rightarrow G$, such that $\phi(w) \neq 1$. This amounts to finding a group $G \in X$ and an $n$-tuple $g_{1}, \ldots, g_{n} \in G$ generating $G$ such that $w\left(g_{1}, \ldots, g_{n}\right) \neq 1$ (and then $\phi$ is defined by sending $x_{i}$ to $\left.g_{i}\right)$. Suppose, given $w$, that there is no $G \in X$ with such an $n$-tuple. Then, for every $G \in X$, and every generating $n$-tuple $\left(g_{1}, \ldots, g_{n}\right) \in G^{n}$, we have $w\left(g_{1}, \ldots, g_{n}\right)=1$. Now, the probability that a random $n$-tuple in $G^{n}$ generates $G$ is the probability that its image in $V^{n}$ spans $V$, where $V=G / \Phi(G)$ is the Frattini quotient of $G$, regarded as a vector space of dimension $\leq n$ over the field with $p$ elements. This probability is at least $\epsilon:=\prod_{i=1}^{n}\left(1-p^{-i}\right)>0$. Thus $P_{G}(w) \geq \epsilon$ for all $G \in X$. By the affirmative answer to Problem 3.2, all the groups $G \in X$ satisfy a common identity $v \neq 1$ (which depends on $w, n$ and $p$ ). This contradiction proves Proposition 3.4.

This argument can be generalized to cases when $X$ consists of finite groups $G$ with the property that $n$ random elements of $G$ generate $G$ with probability bounded away from zero. See [Jaikin-Zapirain and Pyber 2011] and the references therein for the description of such groups and the related notion of positively finitely generated profinite groups.

\section{Acknowledgement}

We would like to acknowledge the referees' very helpful suggestions, which led to some improvements in the paper.

\section{References}

[Abért 2005] M. Abért, "Group laws and free subgroups in topological groups", Bull. London Math. Soc. 37:4 (2005), 525-534. MR 2143732 Zbl 1095.20001

[Aoun 2011] R. Aoun, "Random subgroups of linear groups are free", Duke Math. J. 160:1 (2011), 117-173. MR 2838353 Zbl 1239.20051

[Borel 1983] A. Borel, "On free subgroups of semisimple groups", Enseign. Math. (2) 29:1-2 (1983), 151-164. MR 702738 Zbl 0533.22009

[Breuillard and Gelander 2007] E. Breuillard and T. Gelander, "A topological Tits alternative", Ann. of Math. (2) 166:2 (2007), 427-474. MR 2373146 Zbl 1149.20039 
[Dixon et al. 2003] J. D. Dixon, L. Pyber, Á. Seress, and A. Shalev, "Residual properties of free groups and probabilistic methods", J. Reine Angew. Math. 556 (2003), 159-172. MR 1971144 Zbl 1027.20013

[Epstein 1971] D. B. A. Epstein, "Almost all subgroups of a Lie group are free”, J. Algebra 19 (1971), 261-262. MR 0281776 Zbl 0222.22012

[Jaikin-Zapirain and Pyber 2011] A. Jaikin-Zapirain and L. Pyber, "Random generation of finite and profinite groups and group enumeration”, Ann. of Math. (2) 173:2 (2011), 769-814. MR 2776362 Zbl 1234.20042

[Khukhro 1986] E. I. Khukhro, "Locally nilpotent groups that admit a splitting automorphism of prime order”, Mat. Sb. (N.S.) 130(172):1 (1986), 120-127, 128. MR 847346 Zbl 0608.20025

[Larsen and Shalev 2012] M. Larsen and A. Shalev, "Fibers of word maps and some applications", J. Algebra 354 (2012), 36-48. MR 2879221 Zbl 1258.20011

[Mann 1994] A. Mann, "Finite groups containing many involutions", Proc. Amer. Math. Soc. 122:2 (1994), 383-385. MR 1242094 Zbl 0811.20024

[Neumann 1954] B. H. Neumann, "Groups covered by finitely many cosets", Publ. Math. Debrecen 3 (1954), 227-242. MR 0072138 Zbl 0057.25603

[Neumann 1989] P. M. Neumann, “Two combinatorial problems in group theory”, Bull. London Math. Soc. 21:5 (1989), 456-458. MR 1005821 Zbl 0695.20018

[Szegedy 2005] B. Szegedy, "Almost all finitely generated subgroups of the Nottingham group are free”, Bull. London Math. Soc. 37:1 (2005), 75-79. MR 2105821 Zbl 1073.20022

[Tits 1972] J. Tits, "Free subgroups in linear groups", J. Algebra 20 (1972), 250-270. MR 0286898 Zbl 0236.20032

[Waterhouse 1979] W. C. Waterhouse, Introduction to affine group schemes, Graduate Texts in Mathematics 66, Springer, New York-Berlin, 1979. MR 547117 Zbl 0442.14017

Communicated by Efim Zelmanov

Received 2015-10-29 Revised 2016-05-01 Accepted 2016-05-31

mjlarsen@indiana.edu Department of Mathematics, Indiana University, Rawles Hall, Bloomington, IN 47405-5701, United States

shalev@math.huji.ac.il Einstein Institute of Mathematics,

The Hebrew University of Jerusalem, 91904 Jerusalem, Israel 


\section{Algebra \& Number Theory}

msp.org/ant

\section{EDITORS}

MANAGING EDITOR

Bjorn Poonen

Massachusetts Institute of Technology

Cambridge, USA

\author{
EDITORIAL BOARD CHAIR \\ David Eisenbud \\ University of California \\ Berkeley, USA
}

BOARD OF EDITORS

$\begin{aligned} \text { Dave Benson } & \text { University of Aberdeen, Scotland } & \text { Susan Montgomery } & \text { University of Southern California, USA } \\ \text { Richard E. Borcherds } & \text { University of California, Berkeley, USA } & \text { Shigefumi Mori } & \text { RIMS, Kyoto University, Japan } \\ \text { John H. Coates } & \text { University of Cambridge, UK } & \text { Raman Parimala } & \text { Emory University, USA } \\ \text { J-L. Colliot-Thélène } & \text { CNRS, Université Paris-Sud, France } & \text { Jonathan Pila } & \text { University of Oxford, UK } \\ \text { Brian D. Conrad } & \text { Stanford University, USA } & \text { Anand Pillay } & \text { University of Notre Dame, USA } \\ \text { Hélène Esnault } & \text { Freie Universität Berlin, Germany } & \text { Victor Reiner } & \text { University of Minnesota, USA } \\ \text { Hubert Flenner } & \text { Ruhr-Universität, Germany } & \text { Peter Sarnak } & \text { Princeton University, USA } \\ \text { Sergey Fomin } & \text { University of Michigan, USA } & \text { Joseph H. Silverman } & \text { Brown University, USA } \\ \text { Edward Frenkel } & \text { University of California, Berkeley, USA } & \text { Michael Singer } & \text { North Carolina State University, USA } \\ \text { Andrew Granville } & \text { Université de Montréal, Canada } & \text { Vasudevan Srinivas } & \text { Tata Inst. of Fund. Research, India } \\ \text { Joseph Gubeladze } & \text { San Francisco State University, USA } & \text { J. Toby Stafford } & \text { University of Michigan, USA } \\ \text { Roger Heath-Brown } & \text { Oxford University, UK } & \text { Ravi Vakil } & \text { Stanford University, USA } \\ \text { Craig Huneke } & \text { University of Virginia, USA } & \text { Michel van den Bergh } & \text { Hasselt University, Belgium } \\ \text { Kiran S. Kedlaya } & \text { Univ. of California, San Diego, USA } & \text { Marie-France Vignéras } & \text { Université Paris VII, France } \\ \text { János Kollár } & \text { Princeton University, USA } & \text { Kei-Ichi Watanabe } & \text { Nihon University, Japan } \\ \text { Yuri Manin } & \text { Northwestern University, USA } & \text { Efim Zelmanov } & \text { University of California, San Diego, USA } \\ \text { Philippe Michel } & \text { École Polytechnique Fédérale de Lausanne } & \text { Shou-Wu Zhang } & \text { Princeton University, USA }\end{aligned}$

PRODUCTION

production@msp.org

Silvio Levy, Scientific Editor

See inside back cover or msp.org/ant for submission instructions.

The subscription price for 2016 is US $\$ 290$ /year for the electronic version, and $\$ 485 /$ year (+\$55, if shipping outside the US) for print and electronic. Subscriptions, requests for back issues and changes of subscribers address should be sent to MSP.

Algebra \& Number Theory (ISSN 1944-7833 electronic, 1937-0652 printed) at Mathematical Sciences Publishers, 798 Evans Hall \#3840, c/o University of California, Berkeley, CA 94720-3840 is published continuously online. Periodical rate postage paid at Berkeley, CA 94704, and additional mailing offices.

ANT peer review and production are managed by EditFLow ${ }^{\circledR}$ from MSP.

\section{PUBLISHED BY}

- mathematical sciences publishers

nonprofit scientific publishing

http://msp.org/

() 2016 Mathematical Sciences Publishers 


\section{Algebra \& Number Theory}

Volume $10 \quad$ No. $6 \quad 2016$

Modular elliptic curves over real abelian fields and the generalized Fermat equation $x^{2 \ell}+y^{2 m}=z^{p}$

SAMUELE ANNI and SAMIR SIKSEK

Geometry and stability of tautological bundles on Hilbert schemes of points

DAVID STAPLETON

Anabelian geometry and descent obstructions on moduli spaces

Stefan PATRIKIS, José Felipe Voloch and Yuri G. Zarhin

On the local Tamagawa number conjecture for Tate motives over tamely ramified fields JAY DAIGLE and MATTHIAS FLACH

Heegner divisors in generalized Jacobians and traces of singular moduli

JAN HENDRIK BRUINIER and YINGKUN LI

On 2-dimensional 2-adic Galois representations of local and global fields VYTAUTAS PAŠKŪNAS

A probabilistic Tits alternative and probabilistic identities

Michael LARSEN and ANER SHALEV 